\title{
Analysis and Design of Switched Linear Systems in the Presence of Actuator Saturation and $\mathcal{L}_{2}$ Disturbances *
}

\author{
Liang Lv* Zongli Lin ${ }^{* *}$ Haijun Fang ${ }^{* * *}$ \\ * Department of Automation, Shanghai Jiao Tong University, \\ Shanghai, China. Email: liangup@126.com \\ ** Charles L. Brown Department of Electrical and Computer \\ Engineering, University of Virginia, P.O. Box 400743, Charlottesville, \\ VA 22904-4743, U.S.A.. Email: zl5y@virginia.edu \\ *** MKS Instrument, 100 Highpower Road, Rochester, NY 14623, \\ U.S.A.. Email: haijunfang@ieee.org
}

\begin{abstract}
This paper considers the problem of disturbance tolerance/rejection for a family of linear systems subject to actuator saturation and $\mathcal{L}_{2}$ disturbances. For a given set of linear feedback gains, a given switching scheme and a given bound on the energy of the disturbances, conditions are established in terms of linear or bilinear matrix inequalities under which the resulting switched system is bounded state stable, that is, trajectories starting from a bounded set will remain inside the set or a larger bounded set. With these conditions, both the problem of assessing the disturbance tolerance/rejection capability of the closed-loop system and the design of feedback gain and switching scheme can be formulated and solved as constrained optimization problems. Disturbance tolerance is measured by the largest bound on the disturbances for which the trajectories from a given set remain bounded. Disturbance rejection is measured by the restricted $\mathcal{L}_{2}$ gain over the set of tolerable disturbances. In the event that all systems in the family are identical, the switched system reduces to a single system under a switching feedback law. It will be shown that such a single system under a switching feedback law has stronger disturbance tolerance/rejection capability than a single linear feedback law can achieve.
\end{abstract}

Keywords: actuator saturation, disturbance tolerance, disturbance rejection, $\mathcal{L}_{2}$ gain, set invariance, switched systems.

\section{INTRODUCTION}

The literature on analysis and design of switched systems has been growing rapidly in the recent years (see, for example, Branicky (1994); Cheng (2005); DeCarlo, Branicky, Pettersson \& Lennartson (2000); Liberzon \& Morse (1999); Pettersson (2003, 2004); Sun \& Ge (2005); Wicks, Peleties \& DeCarlo (1998); Xi, Feng, Jiang \& Cheng (2003) and the references therein). Motivated by the results reported in this literature, we consider in this paper the following family of linear systems subject to input saturation and $\mathcal{L}_{2}$ disturbances,

$$
\left\{\begin{array}{l}
\dot{x}=f_{i}(x, u, w), \quad i \in I_{N}:=\{1,2, \cdots, N\}, \\
z=h_{i}(x),
\end{array}\right.
$$

where for each $i \in I_{N}$,

$$
\left\{\begin{array}{l}
\dot{x}=A_{i} x+B_{i} \operatorname{sat}(u)+E_{i} w \\
z=C_{i} x
\end{array}\right.
$$

where $x \in \mathbf{R}^{n}, u \in \mathbf{R}^{m}, z \in \mathbf{R}^{p}$ are respectively the state, input and output of the system, $w \in \mathbf{R}^{q}$ represents the disturbances, and sat $: \mathbf{R}^{m} \rightarrow \mathbf{R}^{m}$ is the

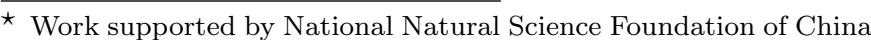
under the Overseas Young Investigator Program (Class B: Overseas Collaboration).
}

vector valued standard saturation function defined as $\operatorname{sat}(u)=\left[\operatorname{sat}\left(u_{1}\right) \operatorname{sat}\left(u_{2}\right) \cdots \operatorname{sat}\left(u_{m}\right)\right]^{\mathrm{T}}$, with $\operatorname{sat}\left(u_{i}\right)=$ $\operatorname{sign}\left(u_{i}\right) \min \left\{\left|u_{i}\right|, 1\right\}$. By defining a controller/supervisor which chooses one of the systems at each time instant based on the measurement of the state and according to an index function, say, $i=\sigma(x)$, a switched system can be defined as

$$
\left\{\begin{array}{l}
\dot{x}=f_{\sigma(x)}(x, u, w) \\
z=h_{\sigma(x)} x
\end{array}\right.
$$

A typical form of the index function is $\sigma(x)=i$ for $x \in \Omega_{i}$ with $\cup_{i=1}^{N} \Omega_{i}=\mathbf{R}^{n}$.

Thus, the control design involves the construction of both feedback gains for individual systems and the index function so that the resulting switched system possesses certain desired performances.

In the absence of the disturbances $w$, a basic design objective is the local asymptotic stability of the resulting switched system with as large a domain of attraction as possible. By utilizing some techniques in dealing with actuator saturation ( $\mathrm{Hu} \& \mathrm{Lin}$ (2001)) and the form of the largest region index function proposed by Pettersson (2003, 2004, 2005), we recently proposed a method for the design of the individual feedback gains and the index function that result in a locally asymptotically stable switched 
system ( $\mathrm{Lv} \&$ Lin (2007)). The design is formulated and solved as a constrained optimization problem with the objective of enlarging the domain of attraction of the resulting stable equilibrium at the origin. It was shown by numerical examples that such a design may result in a domain of attraction larger than that of a switched system designed without taking actuator saturation into account.

In this paper, we will carry out the analysis of and design for the disturbance tolerance/rejection capability of the switched system resulting from the family of systems (2). We will restrict ourselves to a class of $\mathcal{L}_{2}$ disturbances whose energies are bounded by a given value, i.e.,

$$
\mathcal{W}_{\alpha}^{2}:=\left\{w: \mathbf{R}_{+} \rightarrow \mathbf{R}^{q}: \int_{0}^{\infty} w^{\mathrm{T}}(t) w(t) d t \leq \alpha\right\},
$$

for some positive number $\alpha$. For a given set of linear feedback gains, a given index function and a given value of $\alpha$, conditions will be established in terms of linear or bilinear matrix inequalities under which the resulting switched system is bounded state stable. A system is said to be bounded state stable if its trajectories starting from a bounded set will remain inside the set or a larger bounded set. With these conditions, both the problem of assessing the disturbance tolerance/rejection capability of the closed-loop system and the design of feedback gain and switching scheme can be formulated and solved as constrained optimization problems. Disturbance tolerance is measured by the largest bound on the energy of the disturbance, $\alpha^{*}$, for which the trajectories from a given set remain bounded. Disturbance rejection is measured by the restricted $\mathcal{L}_{2}$ gain over $\mathcal{W}_{\alpha^{*}}^{2}$.

An interesting special case of the systems we consider in this paper is the case when all the systems (2) are identical. In this case, the switched system reduces to a single system under a switching linear feedback law. It will be shown that for a single linear system of the form (2), a switching feedback law will result in stronger disturbance tolerance/rejection capability than a single linear feedback law of (Fang, Lin \& Hu (2004); Fang, Lin $\&$ Shamash (2006)). The $\mathcal{L}_{2}$ gain analysis has been one of the most active topics in nonlinear control theory (see, for example, Khalil (2002)). The $\mathcal{L}_{2}$ gain analysis and design for linear systems under actuator saturation has been studied by several authors. A small sample of their works include (Chitour (2001); Fang, Lin \& Hu (2004); Fang, Lin \& Shamash (2006); Hindi (1998); Hu \& Lin (2001); Lin (1997); Nguyen \& Jabbari (1999); Paim, Tarbouriech, Gomes da Silva Jr. \& Castelan (2002); Wada, Oomoto \& Saeki (2004); Xie, Wang, Hao \& Xie (2004); Zhai, Lin \& Kim (2004)). In particular, in our recent work (Fang, Lin \& Hu (2004); Fang, Lin \& Shamash (2006)), we considered the $\mathcal{L}_{2}$ gain analysis and design for a linear system under actuator saturation. The disturbance tolerance capability of the closed-loop system under a given feedback law was assessed, and the linear feedback law that results in a minimized restricted $\mathcal{L}_{2}$ gain was designed.

The remainder of this paper is organized as follows. In Section 2, we state our problem and recall some preliminary materials that will be needed in the development of the results of this paper. Section 3 establishes bounded state stability conditions. Disturbance tolerance and disturbance rejection are addressed in Section 4. Simulation results are presented in Section 5. Section 6 concludes the paper.

\section{PROBLEM STATEMENT AND PRELIMINARIES}

For the family of systems (2), we would like to design a linear feedback law for each individual system in the family and an index function such that the resulting switched system possesses a high degree of disturbance tolerance and a high level of disturbance rejection capabilities.

We will adopt the switching strategy of (Pettersson (2003, 2004)). Such a switching strategy is defined based on some appropriately chosen symmetric matrices $Q_{i} \in \mathbf{R}^{n \times n}, i \in$ $I_{N}$. More specifically, at a given state $x$, the subsystem $i$ will be activated if the quadratic function $x^{\mathrm{T}} Q_{i} x$ is greater or equal to any other $x^{\mathrm{T}} Q_{j} x, j \neq i$. More specifically, this switching scheme is defined by the following index function Pettersson $(2003,2004)$, referred to as the largest region function,

$$
i(x)=\arg \left\{\max _{i \in I_{N}} x^{\mathrm{T}} Q_{i} x\right\} .
$$

Based on the matrices $Q_{i}$ 's, we define the following two sets

$$
\begin{aligned}
\Omega_{i} & =\left\{x \in \mathbf{R}^{n} \mid x^{\mathrm{T}} Q_{i} x \geq 0\right\}, \quad i \in I_{N}, \\
\Omega_{i, j} & =\left\{x \in \mathbf{R}^{n} \mid x^{\mathrm{T}} Q_{j} x=x^{\mathrm{T}} Q_{i} x \geq 0\right\}, \quad i \in I_{N}, j \in I_{N} .
\end{aligned}
$$

Then, a well-defined switched system must satisfy the following properties:

- Covering property: $\Omega_{1} \cup \Omega_{2} \cup \cdots \cup \Omega_{N}=\mathbf{R}^{n}$,

- Switching property: $\Omega_{i, j} \subseteq \Omega_{i} \cap \Omega_{j}, i \in I_{N}, j \in I_{N}$.

The first condition says that there are no regions in the state space where none of the subsystem is activated. The second condition means that a switch from subsystem $i$ to $j$ occurs only for states where the regions $\Omega_{i}$ and $\Omega_{j}$ are adjacent. Consequently, switching occurs on the switching surface $x^{\mathrm{T}} Q_{i} x=x^{\mathrm{T}} Q_{j} x$.

The following two lemmas were established in (Pettersson (2003, 2004)).

Lemma 1. (Covering property). If for every $x \in \mathbf{R}^{n}$,

$$
\theta_{1} x^{\mathrm{T}} Q_{1} x+\theta_{2} x^{\mathrm{T}} Q_{2} x+\cdots+\theta_{N} x^{\mathrm{T}} Q_{N} x \geq 0,
$$

where $\theta_{i}>0, i \in I_{N}$, then $\Omega_{1} \cup \Omega_{2} \cup \cdots \cup \Omega_{N}=\mathbf{R}^{n}$.

Lemma 2. (Switching property). If (5) is satisfied and the largest region function strategy is applied, then the switching property is satisfied.

\section{BOUNDED STATE STABILITY}

We recall a tool from ( $\mathrm{Hu} \&$ Lin (2001)) for expressing a saturated linear feedback $u=\operatorname{sat}(F x)$ on the convex hull of a mixture of the unsaturated control inputs and the auxiliary inputs. For an $F \in \mathbf{R}^{m \times n}$, let $\mathcal{L}(F)=$ $\left\{x \in \mathbf{R}^{n}:\left|f_{i} x\right| \leq 1, i \in I_{m}\right\}$, where $f_{i}$ represents the $i$ th row of matrix $F$. We note that $\mathcal{L}(F)$ represents the region in $\mathbf{R}^{n}$ where $F x$ does not saturate. 
Also, let $\mathcal{V}$ be the set of $m \times m$ diagonal matrices whose diagonal elements are either 1 or 0 . There are $2^{m}$ elements in $\mathcal{V}$. Suppose these elements of $\mathcal{V}$ are labeled as $E_{s}, s \in$ $I_{2^{m}}$. Denote $D_{s}^{-}=I-D_{s}$. Clearly, $D_{s}^{-} \in \mathcal{V}$ if $D_{s} \in \mathcal{V}$. The following lemma is adopted from $\mathrm{Hu} \& \mathrm{Lin}(2001)$.

Lemma 3. Let $F, H \in \mathbf{R}^{l \times n}$. Then, for any $x \in \mathcal{L}(H)$,

$$
\operatorname{sat}(F x) \in \operatorname{co}\left\{D_{s} F x+D_{s}^{-} H x, s \in I_{2^{m}}\right\},
$$

where co stands for the convex hull.

The following theorem deals with the switched system that results from the family of systems (2) and the switching scheme (4).

Theorem 1. If there exist $P_{i}>0, \xi>0, Q_{i}=Q_{i}^{\mathrm{T}}, F_{i} \in$ $\mathbf{R}^{m \times n}, H_{i} \in \mathbf{R}^{m \times n}, \vartheta_{i} \geq 0, \theta_{i}>0$ and $\eta_{i, j}$ such that

$$
\begin{aligned}
& \text { 1. }\left(A_{i}+B_{i}\left(D_{s} F_{i}+D_{s}^{-} H_{i}\right)\right)^{\mathrm{T}} P_{i}+ \\
& \quad P_{i}\left(A_{i}+B_{i}\left(\left(D_{s} F_{i}+D_{s}^{-} H_{i}\right)\right)+\right. \\
& \quad \frac{1}{\xi} P_{i} E_{i} E_{i}^{\mathrm{T}} P_{i}+\vartheta_{i} Q_{i} \leq 0, \quad s \in I_{2^{m}}, i \in I_{N}, \\
& \text { 2. } P_{i}=P_{j}+\eta_{i, j}\left(Q_{j}-Q_{i}\right), \quad i \in I_{N}, j \in I_{N}, \\
& \text { 3. } \theta_{1} Q_{1}+\theta_{2} Q_{2}+\cdots+\theta_{N} Q_{N} \geq 0,
\end{aligned}
$$

and $\mathcal{E}\left(P_{i}, 1+\alpha \xi\right) \cap \Omega_{i} \subset \mathcal{L}\left(H_{i}\right), i \in I_{N}$, then every trajectory of the closed-loop system that starts from inside of $\cap_{i=1}^{N} \mathcal{E}\left(P_{i}, 1\right)$ will remain inside of $\cap_{i=1}^{N} \mathcal{E}\left(P_{i}, 1+\alpha \xi\right)$ for every $w \in \mathcal{W}_{\alpha}^{2}$, as long as no sliding motion occurs or sliding motions only occur along switching surfaces with the corresponding $\eta_{i, j} \geq 0$. If the condition $\mathcal{E}\left(P_{i}, 1+\alpha \xi\right) \cap$ $\Omega_{i} \subset \mathcal{L}\left(H_{i}\right)$ is replaced with $\mathcal{E}\left(P_{i}, \alpha \xi\right) \cap \Omega_{i} \subset \mathcal{L}\left(H_{i}\right)$, then any trajectory starting from the origin will remain inside the region $\cap_{i=1}^{N} \mathcal{E}\left(P_{i}, \alpha \xi\right)$ for every $w \in \mathcal{W}_{\alpha}^{2}$ as long as no sliding motion occurs or sliding motions only occur along switching surfaces with the corresponding $\eta_{i, j} \geq 0$.

\section{DISTURBANCE TOLERANCE AND REJECTION}

\subsection{Disturbance Tolerance}

A fundamental problem to be addressed before the determination of the restricted $\mathcal{L}_{2}$ gain, which measures the disturbance rejection capability, is the assessment of the disturbance tolerance capability of the closed-loop system. The disturbance tolerance capability is measured by the largest bound on the energy of the disturbances, say $\alpha^{*}$, under which the closed-loop trajectories starting from the origin or a given set of initial conditions remain bounded. As the restricted $\mathcal{L}_{2}$ gain will be defined with zero initial conditions, we will only assess disturbance tolerance with zero initial conditions in this section. Disturbance tolerance with a given set of initial conditions can be dealt with similarly by resorting to the first part of Theorem 1 .

As established in Theorem 1, under the three itemized conditions and the condition that $\mathcal{E}\left(P_{i}, \alpha \xi\right) \subset \mathcal{L}\left(H_{i}\right), i \in$ $I_{N}$, the trajectories of the closed-loop system that start from origin will remain inside the region $\cup_{i=1}^{N}\left(\mathcal{E}\left(P_{i}, \alpha \xi\right) \cap\right.$ $\Omega_{i}$ ) for every $w \in \mathcal{W}_{\alpha}^{2}$, if no sliding motion occurs or sliding motions only occur along switching surfaces with the corresponding $\eta_{i, j} \geq 0$.
It is without loss of generality to assume that $\xi=1$ in the above mentioned result. If $\xi \neq 1$, we can multiply both sides of Condition 1 of Theorem 1 with $1 / \xi$ to obtain

$$
\begin{aligned}
\left(A_{i}\right. & \left.+B_{i}\left(D_{s} F_{i}+D_{s}^{-} H_{i}\right)\right)^{\mathrm{T}} \frac{P_{i}}{\xi}+\frac{P_{i}}{\xi}\left(A_{i}+B_{i}\left(D_{s} F_{i}\right.\right. \\
& \left.\left.+D_{s}^{-} H_{i}\right)\right)+\frac{P_{i}}{\xi} E_{i} E_{i}^{\mathrm{T}} \frac{P_{i}}{\xi}+\vartheta_{i} \frac{Q_{i}}{\xi} \leq 0, \quad s \in I_{2^{m}}, i \in I_{N} .
\end{aligned}
$$

Let $\tilde{P}_{i}=P_{i} / \xi$ and $\tilde{Q}_{i}=Q_{i} / \xi$. Then, $\tilde{P}_{i}$ and $\tilde{Q}_{i}$ satisfy all conditions of Theorem 1 with $\xi=1$ and $\mathcal{E}\left(\tilde{P}_{i}, \alpha\right)=$ $\mathcal{E}\left(P_{i}, \alpha \xi\right)$. As a result, the disturbance tolerance capability of the closed-loop system under zero initial conditions can be assessed through solving the following optimization problem,

$$
\sup _{P_{i}>0, Q_{i}=Q_{i}^{\mathrm{T}}, \eta_{i, j}, \vartheta_{i} \geq 0, H_{i}, \theta_{i}>0} \alpha
$$

s.t. (a) $\left(A_{i}+B_{i}\left(D_{s} F_{i}+D_{s}^{-} H_{i}\right)\right)^{\mathrm{T}} P_{i}+P_{i}\left(A_{i}+B_{i}\left(D_{s} F_{i}\right.\right.$ $\left.\left.+D_{s}^{-} H_{i}\right)\right)+P_{i} E_{i} E_{i}^{\mathrm{T}} P_{i}+\vartheta_{i} Q_{i} \leq 0$,

$$
s \in I_{2^{m}}, i \in I_{N}
$$

(b) $P_{i}=P_{j}+\eta_{i, j}\left(Q_{j}-Q_{i}\right), \quad i \in I_{N}, j \in I_{N}$,

(c) $\theta_{1} Q_{1}+\theta_{2} Q_{2}+\cdots+\theta_{N} Q_{N} \geq 0$,

(d) $\mathcal{E}\left(P_{i}, \alpha\right) \cap \Omega_{i} \subset \mathcal{L}\left(H_{i}\right), \quad i \in I_{N}$.

Let $\nu=1 / \alpha$. Then, Constraint (d) is implied by

$$
h_{i, k}\left(P_{i}-\delta_{i} Q_{i}\right)^{-1} h_{i, k}^{\mathrm{T}} \leq \nu, \quad k \in I_{m}
$$

which by Schur complements is equivalent to,

$$
\left[\begin{array}{cc}
\nu & h_{i, k} \\
h_{i, k}^{\mathrm{T}} & \left(P_{i}-\delta_{i} Q_{i}\right)
\end{array}\right] \geq 0, \quad k \in I_{m},
$$

where $\delta_{i}>0$ and $h_{i, k}$ denotes the $k$ th row of $H_{i}$.

Consequently, the optimization problem (7) can be written as the following BMI problem,

$$
\inf _{P_{i}>0, Q_{i}=Q_{i}^{\mathrm{T}}, \eta_{i, j}, \delta_{i}>0, \vartheta_{i} \geq 0, H_{i}, \theta_{i}>0} \nu,
$$

s.t. (a) $\left(A_{i}+B_{i}\left(D_{s} F_{i}+D_{s}^{-} H_{i}\right)\right)^{\mathrm{T}} P_{i}+P_{i}\left(A_{i}+B_{i}\left(D_{s} F_{i}\right.\right.$

$$
\begin{array}{r}
\left.\left.+D_{s}^{-} H_{i}\right)\right)+P_{i} E_{i} E_{i}^{\mathrm{T}} P_{i}+\vartheta_{i} Q_{i} \leq 0, \\
s \in I_{2^{m}}, i \in I_{N},
\end{array}
$$

(b) $P_{i}=P_{j}+\eta_{i, j}\left(Q_{j}-Q_{i}\right), \quad i \in I_{N}, j \in I_{N}$,

(c) $\theta_{1} Q_{1}+\theta_{2} Q_{2}+\cdots+\theta_{N} Q_{N} \geq 0$,

(d) $\left[\begin{array}{cc}\nu & h_{i, k} \\ h_{i, k}^{\mathrm{T}} & \left(P_{i}-\delta_{i} Q_{i}\right)\end{array}\right] \geq 0, \quad k \in I_{m}, i \in I_{N}$.

If we define $P_{N}=P$ and $\eta_{i}=\eta_{j, i}, i \in I_{N-1}$, then, Constraint (b) simplifies to

$$
P_{i}=P-\eta_{i}\left(Q_{N}-Q_{i}\right), \quad i \in I_{N-1},
$$

and consequently, Constraint (a) simplifies to

$$
\text { (a) } \begin{aligned}
( & \left.A_{i}+B_{i}\left(D_{s} F_{i}+D_{s}^{-} H_{i}\right)\right)^{\mathrm{T}}\left(P-\eta_{i}\left(Q_{N}-Q_{i}\right)\right) \\
& +\left(P-\eta_{i}\left(Q_{N}-Q_{i}\right)\right)\left(A_{i}+B_{i}\left(D_{s} F_{i}+D_{s}^{-} H_{i}\right)\right) \\
& +\left(P-\eta_{i}\left(Q_{N}-Q_{i}\right)\right) E_{i} E_{i}^{\mathrm{T}}\left(P-\eta_{i}\left(Q_{N}-Q_{i}\right)\right)
\end{aligned}
$$




$$
\begin{aligned}
& \quad+\vartheta_{i} Q_{i} \leq 0, \quad s \in I_{2^{m}}, i \in I_{N-1}, \\
& \left(A_{N}+B_{N}\left(D_{s} F_{N}+D_{s}^{-} H_{N}\right)\right)^{\mathrm{T}} P \\
& \quad+P\left(A_{N}+B_{N}\left(D_{s} F_{N}+D_{s}^{-} H_{N}\right)\right)+P E_{N} E_{N}^{\mathrm{T}} P \\
& \quad+\vartheta_{N} Q_{N} \leq 0, \quad s \in I_{2^{m}} .
\end{aligned}
$$

In case of switching between only two subsystems, we can set $Q_{1}=Q$ and $Q_{2}=-Q$, where $Q$ is a symmetric matrix. Furthermore, we can, without loss of generality, scale $\theta_{1}=\theta_{2}=1$. This implies that Constraint (c) in (9) is automatically satisfied. The optimization problem (9) then simplifies to

$$
\begin{aligned}
& \inf _{P>0, P-2 \eta Q>0, \eta, Q=Q^{\mathrm{T}}, \vartheta_{1} \geq 0, \vartheta_{2} \geq 0, H_{1}, H_{2}} \nu, \\
& \text { s.t (a) }\left(A_{1}+B_{1}\left(D_{s} F_{1}+D_{s}^{-} H_{1}\right)\right)^{\mathrm{T}}(P-2 \eta Q) \\
& +(P-2 \eta Q)\left(A_{1}+B_{1}\left(D_{s} F_{1}+D_{s}^{-} H_{1}\right)\right) \\
& +(P-2 \eta Q) E_{1} E_{1}^{\mathrm{T}}(P-2 \eta Q)+\vartheta_{1} Q \leq 0, s \in I_{2^{m}}, \\
& \left(A_{2}+B_{2}\left(D_{s} F_{2}+D_{s}^{-} H_{2}\right)\right)^{\mathrm{T}} P \\
& +P\left(A_{2}+B_{2}\left(D_{s} F_{2}+D_{s}^{-} H_{2}\right)\right) \\
& +P E_{2} E_{2}^{\mathrm{T}} P-\vartheta_{2} Q \leq 0, s \in I_{2^{m}}, \\
& \text { (b) }\left[\begin{array}{cc}
\nu & h_{1, k} \\
h_{1, k}^{\mathrm{T}} & P-2 \eta Q-\delta_{1} Q
\end{array}\right] \geq 0, k \in I_{m} \text {, } \\
& {\left[\begin{array}{cc}
\nu & h_{2, k} \\
h_{2, k}^{\mathrm{T}} & P+\delta_{2} Q
\end{array}\right] \geq 0, k \in I_{m} .}
\end{aligned}
$$

\section{2 $\mathcal{L}_{2}$ Gain Estimation}

The restricted $\mathcal{L}_{2}$ gain of the closed-loop system is defined over a set of tolerable disturbances $\mathcal{W}_{\alpha}^{2}, \alpha \in\left(0, \alpha^{*}\right]$ as

$$
\gamma^{*}=\sup _{x(0)=0, w \in \mathcal{W}_{\alpha}^{2} \backslash\{0\}} \frac{\|z\|_{\mathcal{L}_{2}}}{\|w\|_{\mathcal{L}_{2}}},
$$

where $\|\cdot\|_{\mathcal{L}_{2}}$ is the $\mathcal{L}_{2}$ norm of a signal.

The following theorem characterizes the conditions under which the switched linear system has a restricted $\mathcal{L}_{2}$ gain less than or equal to $\gamma$.

Theorem 2. If there exist $P_{i}>0, Q_{i}=Q_{i}^{\mathrm{T}}, F_{i} \in \mathbf{R}^{m \times n}$, $H_{i} \in \mathbf{R}^{m \times n}, \vartheta_{i} \geq 0, \eta_{i, j}$ and $\theta_{i}>0$ such that

$$
\begin{aligned}
& \text { 1. }\left(A_{i}+B_{i}\left(D_{s} F_{i}+D_{s}^{-} H_{i}\right)\right)^{\mathrm{T}} P_{i}+ \\
& \quad P_{i}\left(A_{i}+B_{i}\left(D_{s} F_{i}+D_{s}^{-} H_{i}\right)\right)+P_{i} E_{i} E_{i}^{\mathrm{T}} P_{i}+ \\
& \quad \frac{1}{\gamma^{2}} C_{i}^{\mathrm{T}} C_{i}+\vartheta_{i} Q_{i} \leq 0, \quad s \in I_{2^{m}}, i \in I_{N}, \\
& \text { 2. } P_{i}=P_{j}+\eta_{i, j}\left(Q_{j}-Q_{i}\right), \quad i \in I_{N}, j \in I_{N}, \\
& \text { 3. } \theta_{1} Q_{1}+\theta_{2} Q_{2}+\cdots+\theta_{N} Q_{N} \geq 0,
\end{aligned}
$$

and $\mathcal{E}\left(P_{i}, \alpha\right) \cap \Omega_{i} \subset \mathcal{L}\left(H_{i}\right), i \in I_{N}$, then the restricted $\mathcal{L}_{2}$ gain from $w$ to $z$ over $\mathcal{W}_{\alpha}^{2}$ is less than or equal to $\gamma$, if no sliding motion occurs or sliding motions only occur along switching surfaces with the corresponding $\eta_{i, j} \geq 0$.

By Schur complement, Condition 1 in Theorem 2 is equivalent to

$$
\left[\begin{array}{ccc}
\Gamma_{s, i} & P_{i} E_{i} & C_{i}^{\mathrm{T}} \\
E_{i}^{\mathrm{T}} P_{i} & -I & 0 \\
C_{i} & 0 & -\gamma^{2} I
\end{array}\right] \leq 0 .
$$

where

$$
\begin{aligned}
\Gamma_{s, i}= & \left(A_{i}+B_{i}\left(D_{s} F_{i}+D_{s}^{-} H_{i}\right)\right)^{\mathrm{T}} P_{i} \\
& +P_{i}\left(A_{i}+B_{i}\left(D_{s} F_{i}+D_{s}^{-} H_{i}\right)\right)+\vartheta_{i} Q_{i} .
\end{aligned}
$$

Thus, based on Theorem 2 , the restricted $\mathcal{L}_{2}$ gain can be estimated by solving the following optimization problem,

$$
\begin{gathered}
\inf P_{i}>0, Q_{i}=Q_{i}^{\mathrm{T}}, \eta_{i, j}, \vartheta_{i} \geq 0, H_{i}, \theta_{i}>0 \\
\text { s.t. } \\
\quad \text { (a) }\left[\begin{array}{ccc}
\Gamma_{s i} & P_{i} E_{i} & C_{i}^{\mathrm{T}} \\
E_{i}^{\mathrm{T}} P_{i} & -I & 0 \\
C_{i} & 0 & -\gamma^{2} I
\end{array}\right] \leq 0, s \in I_{2^{m}}, i \in I_{N}, \\
\text { (b) } P_{i}=P_{j}+\eta_{i, j}\left(Q_{j}-Q_{i}\right), \quad i \in I_{N}, j \in I_{N}, \\
\text { (c) } \theta_{1} Q_{1}+\theta_{2} Q_{2}+\cdots+\theta_{N} Q_{N} \geq 0, \\
\text { (d) }\left[\begin{array}{cc}
\frac{1}{\alpha} & h_{i, k} \\
h_{i, k}^{\mathrm{T}} & P_{i}
\end{array}\right] \geq 0, \quad k \in I_{m}, i \in I_{N},
\end{gathered}
$$

In case of switching between only two subsystems, we can set $P_{1}=P, Q_{1}=Q$ and $Q_{2}=-Q$, where $Q$ is a symmetric matrix, and assume, without loss of generality, that $\theta_{1}=\theta_{2}=1$. This implies that Constraint (c) in (12) is automatically satisfied. The optimization problems (12) then simplify to

$$
\inf _{P>0, P-2 \eta Q>0, \eta, Q=Q^{\mathrm{T}}, \vartheta_{1} \geq 0, \vartheta_{2} \geq 0, H_{1}, H_{2}} \nu,
$$

$$
\begin{aligned}
\text { s.t. (a) } & {\left[\begin{array}{ccc}
\Gamma_{s, 1} & (P-2 \eta Q) E_{1} & C_{1}^{\mathrm{T}} \\
E_{1}^{\mathrm{T}}(P-2 \eta Q) & -I & 0 \\
C_{1} & 0 & -\gamma^{2} I
\end{array}\right] \leq 0, s \in I_{2^{m}}, } \\
& {\left[\begin{array}{ccc}
\Gamma_{s, 2} & P E_{2} & C_{2}^{\mathrm{T}} \\
E_{2}^{\mathrm{T}} P & -I & 0 \\
C_{2} & 0 & -\gamma^{2} I
\end{array}\right] \leq 0, \quad s \in I_{2^{m}}, } \\
\text { (b) } & {\left[\begin{array}{cc}
\nu & h_{1, k} \\
h_{1, k}^{\mathrm{T}} P-2 \eta Q-\delta_{1} Q
\end{array}\right] \geq 0, \quad k \in I_{m}, } \\
& {\left[\begin{array}{cc}
\nu & h_{2, k} \\
h_{2, k}^{\mathrm{T}} & P+\delta_{2} Q
\end{array}\right] \geq 0, \quad k \in I_{m}, }
\end{aligned}
$$

\subsection{Solutions of Optimization Problems}

The problem of verifying the existence of the unknown variables solving the above optimization problems are $\mathrm{Bi}$ linear Matrix Inequalities (BMIs) problems, which are NPhard and difficult to solve. However, many algorithms for BMI problems have been proposed on the basis of approximations, heuristics, branch \& bound, or local search. For example, PENOPT offers a commercial solver PENBMI for solving the optimization problems with bilinear matrix inequality constraints. We will use PENBMI to obtain all our numerical results in Section 5.

The above optimization problems can be adapted for the design of feedback gains $F_{i}$ 's. This can be readily done by viewing $F_{i}$ 's as additional optimization parameters.

\section{NUMERICAL EXAMPLES}

Example 1. Let us consider system (2) with 


$$
\begin{aligned}
& A_{1}=\left[\begin{array}{rr}
1 & -5 \\
0 & 0
\end{array}\right], \quad B_{1}=\left[\begin{array}{l}
0 \\
1
\end{array}\right], \quad E_{1}=\left[\begin{array}{l}
0.1 \\
0.1
\end{array}\right], \\
& C_{1}=\left[\begin{array}{ll}
1 & 1
\end{array}\right], \quad F_{1}=\left[\begin{array}{ll}
0 & 1
\end{array}\right], \\
& A_{2}=\left[\begin{array}{ll}
1 & 0 \\
0 & 1
\end{array}\right], \quad B_{2}=\left[\begin{array}{l}
0 \\
1
\end{array}\right], \quad E_{2}=\left[\begin{array}{l}
0.1 \\
0.1
\end{array}\right], \\
& C_{2}=\left[\begin{array}{ll}
1 & 1
\end{array}\right], \quad F_{2}=\left[\begin{array}{ll}
5 & 0
\end{array}\right] .
\end{aligned}
$$

Let $w \in \mathcal{W}_{\alpha}^{2}$. To design a switching law that maximizes the disturbance tolerance capacity of the resulting switched system, we solve the optimization problem (10) and obtain $\nu^{*}=0.3409, \alpha^{*}=2.9336, H_{1}=\left[\begin{array}{lll}-0.3094 & 0.1259\end{array}\right]$, $H_{2}=\left[\begin{array}{ll}3.3443 & -1.3757\end{array}\right], Q=\left[\begin{array}{cc}-0.0422 & 0.0466 \\ 0.0466 & 0.0422\end{array}\right], P_{1}=$ $\left[\begin{array}{cc}11.9804 & 9.9189 \\ 9.9189 & 28.0196\end{array}\right]$ and $P_{2}=\left[\begin{array}{cc}32.8817 & -13.1624 \\ -13.1624 & 7.1183\end{array}\right]$. Plotted in Fig. 1 are the ellipsoids $\mathcal{E}\left(P_{1}, \alpha^{*}\right)$ and $\mathcal{E}\left(P_{2}, \alpha^{*}\right)$, along with a trajectory starting from the origin and under a pulse disturbance of duration $0.2 \mathrm{~s}$ and with a maximum energy $\alpha^{*}$. A zoom in plot of this trajectory is shown in Fig. 2.

We next estimate the restricted $\mathcal{L}_{2}$ gain of the resulting switched system over $\mathcal{W}_{\alpha}^{2}, \alpha \in\left(0, \alpha^{*}\right]$. This can be done by solving the optimization problem (13). Plotted in Fig. 3 is the obtained $\gamma^{*}$ over different value of $\alpha$.

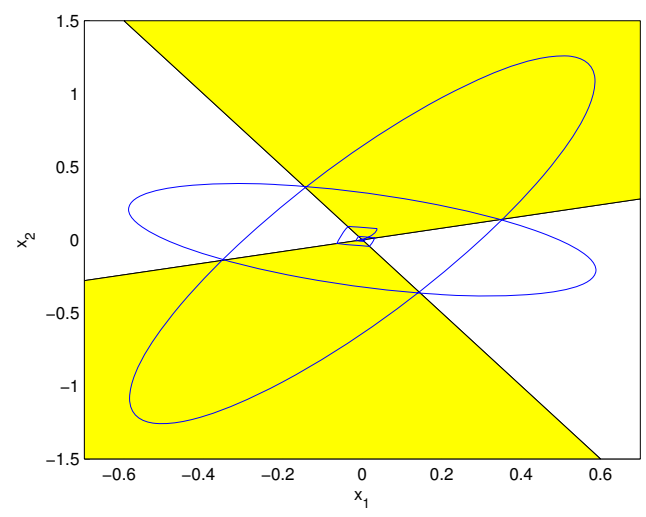

Fig. 1. The ellipsoids $\mathcal{E}\left(P_{1}, \alpha^{*}\right)$ and $\mathcal{E}\left(P_{2}, \alpha^{*}\right)$ and a trajectory under a pulse disturbance with energy $\alpha^{*}$.

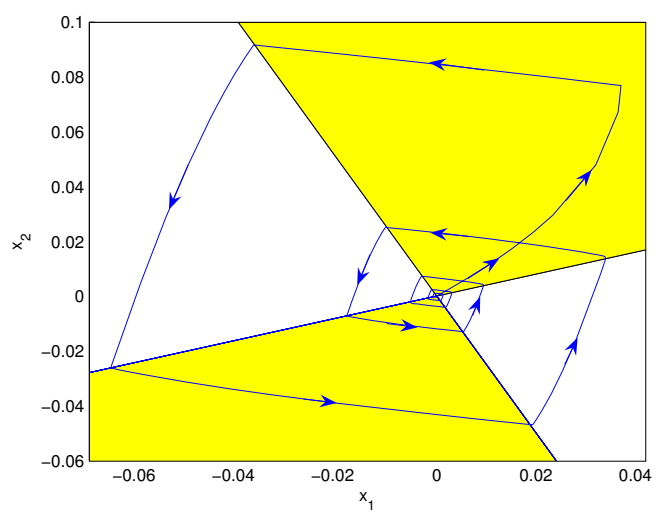

Fig. 2. Zoom in plot of the trajectory shown in Fig. 1.

Example 2. Consider system (2) with

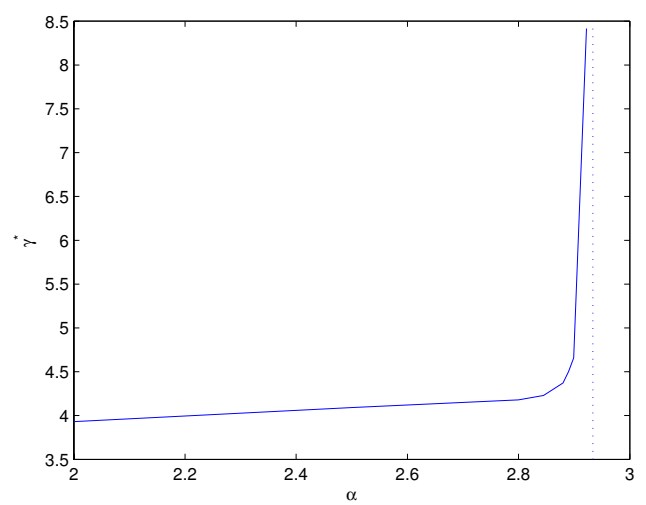

Fig. 3. The restricted $\mathcal{L}_{2}$ gain of the switched system over $\mathcal{W}_{\alpha}^{2}$

$$
\begin{aligned}
& A_{1}=A_{2}=\left[\begin{array}{rr}
0.6 & -0.8 \\
0.8 & 0.6
\end{array}\right], \quad B_{1}=B_{2}=\left[\begin{array}{l}
2 \\
4
\end{array}\right], \\
& E_{1}=E_{2}=\left[\begin{array}{l}
0.1 \\
0.1
\end{array}\right], \quad C_{1}=C_{2}=\left[\begin{array}{ll}
1 & 1
\end{array}\right], \\
& F_{1}=[1.2231-2.2486], \quad F_{2}=\left[\begin{array}{ll}
0.8396 & -1.7221
\end{array}\right] .
\end{aligned}
$$

Also assume that $w \in \mathcal{W}_{\alpha}^{2}$.

We note that two subsystems result from two the different stabilizing feedback gains. For each of these two subsystems, we can use the algorithm in (Fang, Lin \& Hu (2004)) to estimate the restricted $\mathcal{L}_{2}$ gain. In particular, for $\alpha=500$, and the algorithm of (Fang, Lin \& Hu (2004)) results in $\gamma_{1}^{*}=0.1783$ and $\gamma_{2}^{*}=0.1746$.

We next consider the design of a switching law to reduce the restricted $\mathcal{L}_{2}$ gain. To begin with, we solve the optimization problem (10). We obtain a switching law charac-

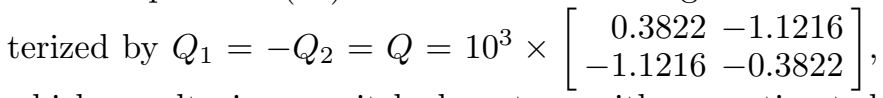
which results in a switched system with an estimated disturbance tolerance capability of $\alpha^{*}=632.8927$.

We next proceed to design a switching law that minimizes the restricted $\mathcal{L}_{2}$ gain of the resulting switched system. To this end, let us set $\alpha=500$. Solving the optimization problem (13), we obtain a switching law characterized by $Q_{1}=-Q_{2}=Q=\left[\begin{array}{rr}-433.9681 & -10.2274 \\ -10.2274 & 433.9681\end{array}\right]$, with $P_{1}=\left[\begin{array}{rr}822.9329 & -688.0880 \\ -688.0880 & 677.0671\end{array}\right], \quad P_{2}=10^{3} \times$ $\left[\begin{array}{rr}1.0000 & -0.6839 \\ -0.6839 & 0.5000\end{array}\right], H_{1}=10^{-51} \times[-0.9690-0.1154]$ and $H_{2}=10^{-43} \times[-0.0459-0.4108]$. This switching law results in a switched system with an estimated restricted $\mathcal{L}_{2}$ gain of $\gamma^{*}=0.1187$. It is clear that $\gamma^{*}<\min \left\{\gamma_{1}^{*}, \gamma_{2}^{*}\right\}$.

To demonstrate the fact that switching improving disturbance rejection capability, we plot in Fig. 4 the outputs of the closed-loop systems, under individual feedback gains $F_{1}$ and $F_{2}$ and the feedback law switching between these two feedback gains, respectively. In the simulation, the initial condition is set to zero and the disturbance is a pulse input of energy $\alpha=625$ starting at $t=0$. The energy of the three different outputs are 4.0995, 4.0888 and 3.8983, respectively. 


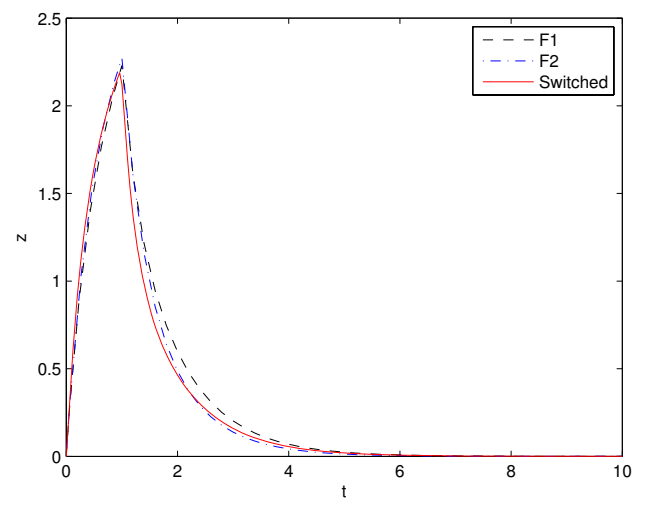

Fig. 4. Trajectories of the closed-loop systems subject to a pulse disturbance with energy $\alpha=625$ and under individual feedback laws and the switched feedback law.

On the other hand, when the actuator saturation does not occur, the closed-loop system resulting from the two individual feedback gains each behaves as a linear system for which the restricted $\mathcal{L}_{2}$ gain can be exactly determined as its $H_{\infty}$ norm. They are $\gamma_{1}^{*}=0.1253$ and $\gamma_{2}^{*}=$ 0.1183. However, in the absence of actuator saturation, the $\mathcal{L}_{2}$ gain of the switched system can be estimated as $\gamma^{*}=0.1072$. This demonstrates the effectiveness of the proposed switching scheme in reducing the $\mathcal{L}_{2}$ gain from the disturbance to the system output, as it results in an $\mathcal{L}_{2}$ gain that is smaller than the $H_{\infty}$ norm of each of the closed-loop systems under the two individual feedback gains.

\section{CONCLUSIONS}

This paper considered the problem of disturbance tolerance/rejection of a switched system resulting from a family of linear systems subject to actuator saturation and $\mathcal{L}_{2}$ disturbances. Design algorithms for both feedback gains for individual systems and the switching scheme were developed and there effectiveness illustrated by numerical examples.

\section{REFERENCES}

A. F. Filippov. Differential Equations with Discontinuous Righthand Sides. Kluwer, Academic Press, 1988.

M. S. Branicky. Stability of switched and hybrid systems. Proc. of the 33rd IEEE Conference on Decision and Control, pages 3498-3503, Lake Buena Vista, FL, 1994.

D. Cheng. Controllability of switched bilinear systems. IEEE Transactions on Automatic Control, volume 50, no. 4, pages 511-515, 2005.

Y. Chitour. On the $\mathcal{L}_{p}$ stabilization of the double integrator subject to input saturation. ESAIM COCV, volume 6 , pages 291-331, 2001.

R. DeCarlo, M. Branicky, S. Pettersson and B. Lennartson. Perspectives and results on the stability and stabilizability of hybrid systems. Proceedings of the IEEE, volume 88, no. 7, pages 1069-1082, 2000.

H. Fang, Z. Lin and T. Hu. Analysis and control design of linear systems in the presence of actuator saturation and $\mathcal{L}_{2}$-disturbances. Automatica, volume 40, no. 7, pages 1229-1238, 2004.
H. Fang, Z. Lin and Y. Shamash. Disturbance tolerance and rejection for linear systems with imprecise knowledge of the actuator input output characteristics. Automatica, volume 42, no. 9, pages 1523-1530, 2006.

H. Hindi and S. Boyd. Analysis of linear systems with saturation using convex optimization. Proc. 37th IEEE Conf. Dec. and Contr., pages 903-908, 1998.

T. Hu and Z. Lin. Control Systems with Actuator Saturation: Analysis and Design. Birkhäuser, Boston, 2001.

H.K. Khalil. Nonlinear Systems. 3rd edition, Prentice Hall, Upper Saddle River, NJ, 2002.

D. Liberzon and A. S. Morse. Basic problems in stability and design of switched systems. IEEE Control System Magazine, volume 19, no. 5, pages 59-70, 1999.

Z. Lin. $H_{\infty}$-almost disturbance decoupling with internal stability for linear systems subject to input saturation. IEEE Trans. Auto. Contr., volume 42, no. 7, pages 992995, 1997.

L. Lv and Z. Lin. Design of switched linear systems in the presence of actuator saturation. IEEE Transactions on Automatic Control, 2008.

T. Nguyen and F. Jabbari. Disturbance attenuation for systems with input saturation: an LMI approach. IEEE Trans. Auto. Contr., volume 44, no. 4, pages 852-857, 1999.

C. Paim, S. Tarbouriech, J.M. Gomes da Silva Jr. and E.B. Castelan. Control design for linear systems with saturating actuators and $l_{2}$-bounded disturbances. Proc. of the 41st IEEE Conf. Dec. Contr., pages 4148-4153, 2002.

S. Pettersson. Synthesis of switched linear systems. Proc. of the 42nd IEEE Conference on Decision and Control, Maui, pages 5283-5288, 2003.

S. Pettersson. Controller design of switched linear systems. Proc. of the 2004 American Control Conference, Boston, Massachusetts, pages 3869-3874, 2004.

S. Pettersson. Synthesis of switched linear systems handling sliding motions. 2005 International Symposium on Intelligent Control, Limassol, Cyprus, pages 18-23, 2005.

Z. Sun and S. Ge. Switched Linear Systems: Control and Design. Springer, Berlin, 2005.

N. Wada, T Oomoto, and M. Saeki. $l_{2}$-gain analysis of discrete-time systems with saturation nonlinearity using parameter dependent Lyapunov function. Proc. of the 43st IEEE Conf. on Dec. Contr., pages 1952-1957, 2004.

M. Wicks, P. Peleties, and R. A. DeCarlo. Switched controller synthesis for the quadratic stabilisation of a pair of unstable linear systems. European Journal of Control, volume 4, no. 2, pages 140-147, 1998.

Z. Xi, G. Feng, Z.P. Jiang and D. Cheng. A switching algorithm for global exponential stabilization of uncertain chained systems. IEEE Transactions on Automatic Control, volume 48, no. 10, pages 1793-1798, 2003.

D. Xie, L. Wang, F. Hao and G. Xie. LMI approach to $\mathcal{L}_{2}$ gain analysis and control synthesis of uncertain switched systems. IEE Proc.-Control Theory Appl., volume 151, no. 1, pages 21-28, 2004.

G. Zhai, H. Lin, and Y. Kim. $\mathcal{L}_{2}$-gain analysis for switched systems with continuous-time and discreteTime subsystems. SICE Annual Conference in Sapporo, Hokkaido Institute of Tecnology, Japan, pages 658663,2004 . 